\title{
RATIONAL COBORDISM OPERATIONS
}

\author{
MARTIN BENDERSKY
}

\begin{abstract}
Rational cobordism operations dual to the right action are studied. The integral operations, and their compositions are computed in terms of these operations. Similar results for $\boldsymbol{B P}$-operations are obtained.
\end{abstract}

Introduction. We study the action of $B P$ cohomology operations on $B P^{*}\left(C P^{\infty}\right)$. The action of the Landweber-Novikov operations on $M U^{*}\left(C P^{\infty}\right)$ is quite simple, so our problem reduces to computing the Quillen idempotent.

In [5], the Quillen map is computed on generators $M_{n}$ of $M U_{*}(M U)$, for small values of ${ }^{*}$. (These generators are related to the duals of the Landweber-Novikov operations by the canonical anti-isomorphism.)

We formalize this procedure by introducing rational operations $\rho_{F}$, which are related to the right action by duality (2.1). These operations behave well with respect to the Quillen map, so the difficulty is reduced to computing $\rho_{F}$ on $M U Q^{*}\left(C P^{\infty}\right)$. This allows us to work entirely in cohomology, where the composition laws for $\rho_{F}$ are simple. In particular the computation of the action of a composition of operations on $B P^{*}\left(C P^{\infty}\right)$ is no more difficult than computing the action of a single operation (2.4).

I would like to thank M. Mimura for posing the question which led to this paper.

1. Preliminaries on $M U$ and $B P$. We adopt the notation of [1], [2] and [6]. $M U_{*}$ is a polynomial algebra on even dimensional generators.

$$
M U^{-*}=M U_{*}=Z\left[x_{1}, x_{2}, \ldots\right],\left|x_{i}\right|=2 i .
$$

$M U_{*}(M U)$ is a polynomial algebra over $M U_{*}$.

$$
M U_{*}(M U)=M U_{*}\left[b_{1}, b_{2}, \ldots\right], \quad\left|b_{i}\right|=2 i .
$$

We may also choose generators $M_{i}$ which are related to $b_{i}$ by the canonical anti-isomorphism $c\left(b_{i}\right)=M_{i}$.

Over the rationals, it is possible to choose generators, $m_{i}$ for $M U Q_{*}$ so that the right action is given by

$$
\eta_{R}\left(m_{i}\right)=\sum_{a+d=i} m_{a}(M)_{d}^{a+1}
$$

where $M=\sum_{i \geqslant 0} M_{i}$ and $(M)_{d}^{a+1}$ denotes the term of degree $2 d$ in $(M)^{a+1}$.

Similarly, for a fixed prime $p$

Received by the editors April 25, 1977.

AMS (MOS) subject classifications (1970). Primary 55B20, 55G25.

C. American Mathematical Society 1978 


$$
B P^{-*}=B P_{*}=Q_{p}\left[v_{1}, v_{2}, \ldots\right]
$$

where $\left|v_{i}\right|=2\left(p^{i}-1\right)$, and $Q_{p}$ denotes the integers localized at $p$.

$$
B P_{*}(B P)=B P_{*}\left[t_{1}, t_{2}, \ldots\right], \quad\left|t_{i}\right|=2\left(p^{i}-1\right) \text {. }
$$

We may choose generators for $B P Q_{*}$ so that

$$
B P Q_{*}=Q\left[m_{1}, m_{2}, \ldots\right], \quad\left|m_{i}\right|=2\left(p^{i}-1\right)
$$

and the right action is given by

$$
\eta_{R}\left(m_{i}\right)=\sum_{a+d=i} m_{a} t p^{a}
$$

The Quillen map $\varepsilon: M U Q_{p} \rightarrow B P$ is a map of ring spectra, and induces natural transformations

$$
\varepsilon^{*}: M U Q_{p}^{*}(X) \rightarrow B P^{*}(X)
$$

and

$$
\varepsilon_{*}: M U Q_{p *}(X) \rightarrow B P_{*}(X) .
$$

Over the rationals we have, for $X=S^{0}$

$$
\varepsilon_{*}\left(m_{i}\right)= \begin{cases}m_{r}, & i=p^{r}-1, \\ 0, & i \neq p^{r}-1, \text { any } r .\end{cases}
$$

The ring of cohomology operations in $M U^{*}(M U)$ is the completed tensor product $M U^{*} \hat{\otimes} S$ where $S$ is the free group generated by elements $S_{F}$. ( $F=\left(f_{1}, f_{2}, \ldots\right)$ runs over all sequences of nonnegative integers almost all of which are zero.) $\left\{S_{F}\right\}$ is the basis dual to $\left\{b^{F}=b_{1}^{f_{1}} b_{2}^{f_{2}} \ldots\right\} . B P^{*}(B P)$ is the completed tensor product $B P^{*} \hat{\otimes} R$ where $R$ is the free $Q_{p}$ module generated by $r_{F}$, the dual basis to $t^{F}$.

Let $E$ be a spectrum satisfying the flatness condition [1]. For $x \in E_{*}(X)$, $\psi(x)=\sum e_{i} \otimes x_{i}, e_{i} \in E_{*}(E), x_{i} \in E_{*}(X), r \in E^{*}(E)$, and $u \in E^{*}(X)$ we have the formula of Adams [1, p. 73].

$$
\langle r(u), x\rangle=\sum\left\langle r, e_{i}\left\langle u, x_{i}\right\rangle\right\rangle \text {. }
$$

For $X=C P^{\infty}, E=M U$ or $B P, E^{*}\left(C P^{\infty}\right)=E^{*}[[u]], u \in E^{2}\left(C P^{\infty}\right)$. So, in this case the action of $E^{*}(E)$ on $E^{*}(X)$ is determined by the coaction, $\psi$, via (1.4). Furthermore, we may choose $u$ in $B P$ and $M U$ so that $\varepsilon^{*}(u)=u$.

In the case of $E=M U$ we have

$$
S_{F}(u)= \begin{cases}u^{i+1}, & F=\Delta_{i}, \\ 0, & F \neq \Delta_{i}\end{cases}
$$

where $\Delta=(0, \ldots, 1, \ldots), 1$ in the $i$ th place. The coaction is given by the formula

$$
\psi\left(u_{i}\right)=\sum_{a+d=i}(b)_{d}^{a} \otimes u_{a}
$$

where $\left\{u_{k}\right\}$ is the basis dual to $\left\{u^{k}\right\}$. 
2. Rational operations. The formal properties of the right action dualize to convenient composition laws in cohomology. These operations also behave well with respect to the Quillen map. Specifically let $E$ denote $M U Q$ or $B P Q$.

THEOREM 2.1. There are operations $\rho_{F}^{E} \in E^{|F|}(E)\left(|F|=\sum 2 i f_{i}\right.$ for $E=$ $M U Q,|F|=\sum 2\left(p^{i}-1\right) f_{i}$ for $\left.E=B P Q\right)$ such that

(i) $F^{*}(F)=F^{*} \hat{\otimes} P$ where $P$ is the $Q$ vector space generated by $\left\{\rho_{F}^{E}\right\}$.

(ii) $\Delta\left(\rho_{F}^{E}\right)=\Sigma_{F_{1}+F_{2}=F} \rho_{F_{1}}^{E} \otimes \rho_{F_{2}}^{E}$.

(iii)

$$
\rho_{F}^{E}\left(m^{G}\right)= \begin{cases}1, & F=G \\ 0, & F \neq G\end{cases}
$$

where $m^{G}=m_{1}^{g_{1}} m_{2}^{g_{2}} \ldots$

(iv) $\rho_{F}^{E} \circ \rho_{D}^{E}=0$ if $F \neq(0,0, \ldots)=(0), \rho_{(0)}^{E} \circ \rho_{D}^{E}=\rho_{D}^{E}$.

Proof. We prove 2.1 for $F=M U Q$. The proof for $B P Q$ is similar.

Set $d_{k}$ equal to $\eta\left(m_{k}\right)$. (1.1) implies

$$
M U Q_{*}(M U)=M U Q_{*}\left[d_{1}, d_{2}, \ldots\right]
$$

then $d^{F}=\eta\left(m^{F}\right)$ is an $M U Q_{*}$ basis for $M U Q_{*}(M U)$. Define $\left\{\rho_{F}=\rho_{F}^{M U}\right\}$ to be the basis of $M U Q^{*}(M U)$ dual to $\left\{d^{F}\right\}$. (i) and (ii) are immediate. From (1.4), with $X=S^{0}$ we have

$$
\left\langle\rho_{F}\left(m^{G}\right), 1\right\rangle=\left\langle\rho_{F}, d^{G}\right\rangle
$$

and (iii) follows.

To prove (iv) we use the formula

$$
\psi\left(d^{F}\right)=1 \otimes_{M U Q_{*}} d^{F} \quad([1, \text { page } 64]) .
$$

Hence

$$
\left\langle\rho_{F}^{\circ} \rho_{D}, d^{G}\right\rangle=\left\langle\rho_{F}, 1 \cdot\left\langle\rho_{D}, d^{G}\right\rangle\right\rangle
$$

which is 0 unless $F=(0,0, \ldots)$. For $F=(0,0, \ldots)$

$$
\left\langle\rho_{(0)} \circ \rho_{D}, d^{G}\right\rangle= \begin{cases}0, & G \neq D \\ 1, & G=D\end{cases}
$$

and (iv) follows.

REMARKS. (1) $\rho_{(0)}^{E}$ is not $1 \in E^{0}(E)$ in fact the counit, $\varepsilon$ satisfies $\varepsilon\left(d^{F}\right)=$ $m^{F}$. This implies $1=\sum m^{F} \rho_{F}$.

(2) 2.1 generalizes to ring spectra satisfying the following conditions.

(a) $\pi_{*}(E)=0, *<0$.

(b) $\pi_{*}(E)$ is a finitely generated vector space over the rationals, with $\pi_{0}(E)=Q$, generated by the unit.

Then, with respect to a basis $\left\{x_{i}\right\}$ (with $1=x_{0}$ ) for $E_{*}$, one may choose generators $\rho_{i}$, dual to $\eta\left(x_{i}\right)$. This follows from the collapsing of the AtiyahHirzebruch spectral sequence. The $\rho_{i}$ 's satisfy (i), (iii) and (iv).

If $E_{*}$ is a polynomial algebra then we may choose generators satisfying (ii). 
We now restrict our attention to $B P Q_{*}$ for the remainder of this section, and denote $\rho_{F}^{B P}$ by $\rho_{F}$.

Let $\boldsymbol{F}=\left(f_{1}, f_{2}, \ldots\right), G=\left(g_{1}, g_{2}, \ldots\right)$ be sequences of nonnegative integers, almost all of which are zero. Define elements $W_{F, G} \in B P Q^{*}$ by the formula

$$
\sum W_{F, G} t^{G}=\prod\left(\sum_{a+b=n} m_{a} t_{b}^{p^{a}}\right)^{f_{n}}=d^{F} .
$$

PRoposition 2.2. $r_{G}=\Sigma_{F} W_{F, G} \rho_{F}$.

Proof. This is dual to (1.2).

For $F=\left(f_{1}, f_{2}, \ldots\right)$, and a prime $p$ define

$$
p(F)=f_{1}+p f_{2}+p^{2} f_{3}+\cdots, \quad s(F)=\left(f_{2}, f_{3}, \ldots\right) .
$$

If $G=\left(g_{1}, g_{2}, \ldots\right)$, then the binomial coefficient $\left(\begin{array}{c}F \\ G\end{array}\right)$ is defined to be

$$
\left(\begin{array}{l}
f_{1} \\
g_{1}
\end{array}\right)\left(\begin{array}{l}
f_{2} \\
g_{2}
\end{array}\right) \ldots
$$

For $I \subset B P Q^{*},(I)$ denotes the ideal $(I) \cdot B P Q^{*}(B P) \subset B P Q^{*}(B P)$. With this notation, we may specialize 2.2 .

CoROllaRY 2.3. (a) $r_{(n)}=\sum_{p(F)=n}\left(\begin{array}{c}F+G \\ F\end{array}\right) m^{s(F)+G} \rho_{F+G}$ where $(n)=(n, 0$, $0, \ldots)$.

(b) $r_{F}=\rho_{F} \bmod \left(m_{1}, m_{2}, \ldots\right)$.

Theorem 2.1 implies we can write compositions of operations, $r_{(n)}$ directly in terms of the basis $\left\{\rho_{F}\right\}$, and thereby obtain relations of the Adem type.

Let $N=\left(n_{k}, \ldots, n_{2}, n_{1}\right)$ be a sequence of nonnegative integers. We define

$$
r^{N}=r_{\left(n_{k}\right)} \circ \cdots \circ r_{\left(n_{2}\right)} \circ r_{\left(n_{1}\right)} \text {. }
$$

For $i=1, \ldots, k$ let $F_{i}=\left(f_{1}^{i}, f_{2}^{i}, \ldots\right)$ be a sequence of almost all zero, nonnegative integers. Let $T$ denote $\left(F_{k}, \ldots, F_{1}\right)$. Fix a prime $p$. Then we define $p(T)=\left(p\left(F_{k}\right), \ldots, p\left(F_{1}\right)\right),|T|=F_{k}+\cdots+F_{1}$. For $G=\left(g_{1}\right.$, $\left.g_{2}, \ldots\right)$

$$
C(G, T)=\prod_{q=0}^{k-1}\left(\begin{array}{c}
G+F_{1}+S\left(\sum_{i=1}^{q} F_{i}\right)-\sum q F_{i} \\
F_{q+1}
\end{array}\right)
$$

where, by convention, $\Sigma_{1}^{0}=0$. With this notation, we have the following.

Proposition 2.4. $r^{N}=\Sigma_{p(T)=N} C(G, T) m^{\left(G+F_{1}+S|T|-|T|\right)} \rho_{G+F_{1}}$.

Proof. This is a straightforward induction using (2.1) and (2.3).

EXAMPLES.

$$
r_{(1)} \overbrace{\cdots \cdots \circ}^{s} r_{(1)}=s ! r_{(s)} \bmod \left(v_{1}, v_{2}, \ldots\right) .
$$

(b) $\quad r_{(1,1)}=r_{(2)} \circ r_{(p)}+\left(\begin{array}{c}p+2 \\ 2\end{array}\right)\left(\begin{array}{l}p \\ 2\end{array}\right) r_{(p+2)} \bmod \left(v_{1}, v_{2}, \ldots\right)$. 
(c) If $m, n<p$ then $r_{(m)} \circ r_{(n)}=r_{(n)} \circ r_{(m)}$. (Compare [6].)

Proof. For part (a), $T$ must be the sequence ((1), ., (1)). So $S(|T|)=0$ and $\Sigma q F_{i}=(q)$. The only term in $r^{(1, \ldots, 1)}$ not zero $\bmod \left(m_{1}, \ldots\right)$ is the term with $G=(s-1)$. In this case, $C(G, T)=s$ !. Part (a) now follows from 2.3 (b), and the fact that $\left(v_{1}, v_{2}, \ldots\right) \otimes Q=\left(m_{1}, m_{2}, \ldots\right)$ [3]. Parts (b) and (c) are similar.

REMARKs. (1) R. Zahler has obtained an Adem relation for compositions of the elements $r_{\left(p^{i}\right)}$.

(2) The ideal $\left(v_{1}, v_{2}, \ldots\right)$ is not invariant [4]. So (a) and (b) are only correct on classes $x \in B P^{*}(X)$, not on classes defined $\bmod \left(v_{1}, v_{2}, \ldots\right)$.

3. Applications to $C P^{\infty}$. The behavior of $\rho_{F}$ with respect to the Quillen map is particularly simple. Let $\Delta_{i}$ denote $(0, \ldots, 1,0, \ldots)$ with 1 in the $i$ th place.

Proposition 3.1. Suppose $H=\Sigma f_{r} \Delta_{p^{\prime-1}}$ and $\rho_{H}^{M U}(u)=\sum x_{i, H} u^{i}, x_{i, H} \in$ $M U Q^{*}$. Then $\rho_{F}^{B P}=\sum \varepsilon_{*}\left(x_{i, H}\right) u^{i}$, where $\varepsilon_{*}$ is as in (1.3), and $u$ is defined in $\S 1$.

Proof.

$$
\psi^{M U}\left(u_{k}\right)=\Sigma x_{i, H} d^{H} \otimes u_{1}+\text { (other terms). }
$$

By the naturality of the coaction, the naturality of $\eta$, and (1.3)

$$
\psi^{B P}\left(u_{k}\right)=\Sigma \varepsilon\left(x_{i, H}\right) d^{F} \otimes u_{1}+\text { (other terms) }
$$

where (other terms) does not involve $u_{1} \cdot 3.1$ now follows from (1.4).

We now consider operations on $B P^{*}\left(C P^{n}\right)=B P^{*}[u] / u^{n+1}=0$. In particular we consider $r_{(n-k)}$ acting on $u^{k}$. In this case we need only compute $r_{(n-k)} \bmod \left(v_{1}, v_{2}, \ldots\right)$ i.e. $\rho_{(n-k)}^{B P}$.

By 3.1 we must compute $\rho_{(n-k) \Delta_{p-1}}^{M U}$. By the Cartan formula it suffices to compute $\rho_{(n-k) \Delta_{p-1}}^{M U}(u)$. For the remainder of this section $\rho_{*}$ will denote $\rho_{*}^{M U}$.

We wish to compute the coefficients $x_{i, l} \in M U Q^{*}$ in the expansion

$$
\rho_{l \Delta_{p-1}}(u)=\Sigma_{i} x_{i, l} u^{i}, \quad\left(\left|x_{i, l}\right|=2[l(p-1)-i+1]\right) .
$$

Set $y_{l}=\Sigma_{i} x_{i, l}, Y(z)=\sum y_{l} z^{l}\left(z\right.$ an indeterminate), and $m=1+m_{1}+m_{2}$ $+\cdots$.

THEOREM 3.2. $y_{l}$ is recursively determined by the formula

$$
z(Y(z))^{p}+Y(z)-m=0 .
$$

In particular, for $p=2, Y(z)=(-1+\sqrt{1+4 m z}) / 2 z$.

Proof.

$$
y_{l}=\sum_{i}\left\langle\rho_{l \Delta_{p-1}}(u), u_{i}\right\rangle=\sum_{i}\left\langle\rho_{l \Delta_{p-1}},(b)_{i-1}^{1}\right\rangle .
$$

Hence $b=\Sigma y_{l} d^{l \Delta_{p-1}}+\left(\right.$ other terms). From $(1.1), \eta(m)=\Sigma m_{a}(M)^{a+1}$. Applying the canonical anti-isomorphism to both sides, we obtain $m=$ $\Sigma \eta\left(m_{a}\right)(b)^{a+1}$. For $l>0$,

$$
0=\left\langle\rho_{l \Delta_{p-1}}, m\right\rangle=\left\langle\rho_{l \Delta_{p-1}}, b+b^{p} \eta\left(m_{p-1}\right)\right\rangle
$$


and for $l=0$,

$$
m=\left\langle\rho_{l \Delta_{p-1}}, b+b^{p} \eta\left(m_{p-1}\right)\right\rangle .
$$

So we have the recursion formula

$$
m=\left(\sum y_{l} d^{l \Delta_{p-1}}\right)+\left(\sum y_{l} d^{l \Delta_{p-1}}\right) d^{\Delta_{p-1}} .
$$

Setting $d^{\Delta_{p-1}}$ equal to the indeterminate $z$ proves 3.2.

We conclude with some examples for $p=2$.

Let $R=\Sigma_{l \geqslant 0} r_{(l)}$. The Cartan formula implies

$$
\begin{gathered}
R\left(u^{k}\right)=R(u)^{k} \quad \text { in } B P^{*}\left(C P^{n}\right) \\
\rho_{(l)}^{M U}(u)=\Sigma_{i} x_{i, l} u^{i}=x_{l+1, l} u^{l+1} \bmod \left(m_{1}, m_{2}, \ldots\right)
\end{gathered}
$$

and

$$
Y(z)=\Sigma y_{l} z^{\prime}=\Sigma x_{l+1, l} z^{\prime}=\frac{-1+\sqrt{1+4 z}}{2 z} \bmod \left(m_{1}, m_{2}, \ldots\right) .
$$

From 3.1 we have for the prime 2

$$
R\left(u^{k}\right)=u^{k}\left(\frac{-1+\sqrt{1+4 u}}{2 u}\right)^{k} \bmod \left(v_{1}, v_{2}, \ldots\right)
$$

where $R=r_{(0)}+r_{(1)}+\cdots$.

\section{REFERENCES}

1. J. F. Adams, Lectures on generalized cohomology, Lecture Notes in Math., vol. 99, SpringerVerlag, Berlin, 1969.

2. Stable homotopy, and generalized homology, University of Chicago Press, Chicago, 1974.

3. M. Hazewinkel, Constructing formal groups $I$ over $Z_{(p)}$-algebras, Report 7119 , Netherlands School of Economics, Econometric Institute.

4. P. S. Landweber, Annihilator ideals, and primitive elements in complex bordism, Illinois J. Math. 17 (1973), 273-283.

5. A. Liulevicius, On the algebra $B P_{*}(B P)$, Lecture Notes in Math., vol. 249, Springer-Verlag, Berlin, 1971.

6. R. Zahler, The Adams-Novikov spectral sequence for the spheres, Ann. of Math. 96 (1972), 480-504.

Department of Mathematics, University of Washington, Seattle, Washington 98195 